\title{
Responsabilidad Social para la Interacción Comunitaria en contextos universitarios: Una aproximación teórica
}

\section{Social Responsibility for Community Interaction in university contexts: A theoretical approach}

\author{
Marelys Castro \\ marelysunesr@gmail.com \\ Universidad Nacional Experimental Simón Rodríguez \\ Grupo de Investigación CIENCIAMATRIA \\ Venezuela \\ Josía Isea \\ josiaisea@gmail.com \\ Universidad Nacional Experimental Francisco de Miranda \\ Grupo de Investigación CIENCIAMATRIA \\ Venezuela \\ https://orcid.org/0000-0001-8921-6446
}

Recibido: 01/03/2018

Aprobado: 21/04/2018

\section{RESUMEN}

La educación universitaria está urgida de estudios interpretativos y subjetivos que permitan interpretar, comprender y generar la responsabilidad social para la función interacción comunitaria en contextos universitarios. El presente estudio se planteó como propósito central es develar un constructo teórico que fundamente la responsabilidad social en pro de la función interacción comunitaria en contextos universitarios: Caso: UNESR (Universidad Nacional Experimental Simón Rodríguez. La investigación fue de corte cualitativo. El procesamiento interpretativo analítico se produce desde el planteamiento de la teoría fundamentada propuesta por Glaser y Strauss (1967) en la cual, se utiliza el análisis de contenido para la codificación y la comparación constante para la categorización por agrupamiento, bajo el principio inductivo. A partir de las diversas categorías emergentes que surgieron, estableciendo conexiones entre ellas lo cual permitió establecer un hilo conductor para ir 


\section{CIENCIAMATRIA \\ Revista Interdisciplinaria de Humanidades, Educación, Ciencia y Tecnología \\ Año V. Vol. V. №9. Julio - Diciembre 2019 \\ Hecho el depósito de ley: pp201602FA4721 \\ ISSN-L: 2542-3029; ISSN: 2610-802X \\ Universidad Nacional Experimental Francisco de Miranda (UNEFM). Santa Ana de Coro. Venezuela}

Marelis Castro; Josía Isea

construyendo o ir generando las representaciones sociales que coadyuvaron a precisar que generar la responsabilidad social para la interacción comunitaria en contextos universitarios pasa por reconocer como núcleos del saber o ejes articulantes: La necesidad que la universidad asuma una seria posición critico - reflexiva de su accionar en pro de un desarrollo social, para luego gestar una verdadera transformación de marcado carácter socialista desde una praxis educativa socio implicadora y comprometida con el accionar comunitario.

Descriptores: Responsabilidad Social; Interacción comunitaria; Educación Universitaria; Universidad; cultura universitaria.

\section{SUMMARY}

The university education is urgent of interpretative and subjective studies that allow to interpret, understand and generate social responsibility for the community interaction function in university contexts. The present study was proposed as a central purpose is to unveil a theoretical construct that bases social responsibility in favor of the community interaction function in university contexts: Case: UNESR (Simón Rodríguez National Experimental University.) The research was qualitative. it occurs from the approach of the grounded theory proposed by Glaser and Strauss (1967) in which content analysis is used for coding and constant comparison for categorization by grouping, under the inductive principle. Emerging categories that emerged, establishing connections between them, which allowed to establish a common thread to build or generate social representations that helped to clarify that generating social responsibility for community interaction in university contexts is to recognize as a eos del saber o articulantes axes: The need for the university to assume a serious critical position - reflective of its actions in favor of social development, to then create a true transformation of a marked socialist character from an educational practice that is both involved and committed to the action community

Descriptors: Social Responsibility; Community interaction; University education; College; university culture

\section{A MODO INTRODUCTORIO}

En los tiempos actuales, se exige de una educación que responda a las necesidades sentidas de las comunidades, reimpulsando el aparato productivo de la misma, la revalorización del saber tácito de cada uno de los habitantes, el reconocimiento de la identidad cultural, el trabajo en equipo mancomunado, coadyuvando a mejorar la calidad de vida de todos y todas. En este sentido, el proceso de transformación de la 
Marelis Castro; Josía Isea

Educación Universitaria, y en especial, en las Universidades Nacionales en Venezuela es una necesidad impostergable. Es necesario nuevos métodos de acercamiento con las comunidades, en donde las universidades sean vistas como una institución con y para las comunidades, y las comunidades como ese espacio para confrontar ideas, aprehender del saber cotidiano, establecer la relación de ayuda; en una sociedad cada día más cambiante, que exige la articulación de ambos procesos educativos (Isea, 2018).

A esta realidad, a pesar de los grandes esfuerzos por parte del Estado y de las propias políticas institucionales de la Universidad Venezolanas pudieran no escapar las mismas; pues pareciera que aún no logran concretar una labor extensionista o de interacción comunitaria con principios liberadores hacia la responsabilidad social cono eje contribuyente en el progreso integral del pueblo venezolano. Al respecto respecto de lo anterior, señalan Arias y Sarmiento (2017) que:

En estos últimos años se han multiplicado las actividades en torno a la problemática económica, social y ambiental a nivel global y el reto de la sustentabilidad del planeta. En ellas, el compromiso de las empresas sobresale como un factor decisivo. En este sentido, se observa a nivel mundial un notable esfuerzo por incorporar la Responsabilidad Social de las Empresas (RSE), para avanzar hacia la sustentabilidad, mediante actividades básicas para solventar las dificultades que enfrentan los grupos humanos; es decir, como un objetivo clave de la productividad y la mejora de la calidad de vida de los ciudadanos a nivel global.

Este aspecto es transversal a todos los agentes que contribuyen al desarrollo de la sociedad, en especial, las universidades; ya que ellos tienen la misión de formar desde una consciencia social que permita el desarrollo del entorno para el mejoramiento de la calidad de vida del ciudadano.

La investigación parte de conversaciones informales, no estructuradas con docentes y estudiantes, se ha focalizado la percepción que la interacción comunitaria en la actualidad se enfoca al llamado "servicio comunitario", es allí donde los docentes manifiestan realizar interacción comunitaria (extensión universitaria), siendo esta apenas una arista en donde se puede tener acceso a las comunidades, pero es de 


\section{CIENCIAMATRIA \\ Revista Interdisciplinaria de Humanidades, Educación, Ciencia y Tecnología \\ Año V. Vol. V. №9. Julio - Diciembre 2019 \\ Hecho el depósito de ley: pp201602FA4721 \\ ISSN-L: 2542-3029; ISSN: 2610-802X \\ Universidad Nacional Experimental Francisco de Miranda (UNEFM). Santa Ana de Coro. Venezuela}

Marelis Castro; Josía Isea

recordar que el servicio comunitario es una ley de cumplimiento obligatorio y está confinada a un máximo de 120 horas, ante lo cual, la interacción comunitaria no puede quedarse relegada al hecho de cumplir con este requisito, es necesario tener una mayor participación activa y percibir la interacción comunitaria como un medio para la gestión del conocimiento en pro de establecer una responsabilidad social que permita a la Universidad, promover cambios en la comunidad mediante el empoderamiento del conocimiento.

Por consiguiente, desde la percepción del colectivo docente y estudiantil, pareciera existir la concepción errónea de que la responsabilidad social universitaria y la interacción comunitaria es el llamado servicio comunitario; y éste es solamente abordado como un requisito que deben cumplir el estudiantado para obtener su titulación, es decir prácticamente como un obstáculo para su profesionalización y no como parte de un querer, un saber y un poder de participación que les corresponde como un derecho y un deber pleno de responsabilidad socio comunitaria.

Lo anterior, se evidencia empíricamente, desde la convivencia de los investigadores con estudiantes y docentes en el recinto de la Universidad Nacional Experimental Simón Rodríguez, así como en experiencias en el abordaje de interacción comunitaria y servicio comunitario; básicamente, se pudo observar que los primeros a menudo inscribían el servicio comunitario pero no lo culminaban o enmarcaban los proyectos en comunidades diferentes a las que residen, esto aparentemente sin una razón de peso, los docentes muestran apatía en cuanto a generar proyectos desde las diferentes unidades curriculares que involucren la comunidad, y en general se aprecia una falta de compromiso y convicción por accesar a la misma en pro de gestar acciones colectivas que generen impacto en el bienestar humano del binomio Universidad Comunidad. Lo anteriormente señalado, denota lo que no deber ser las competencias del docente universitario o estudiante universitarios con al sentido social, ya que según Isea; Briceño y Mayorga (2019:23) "un proyecto (...) de tipo Investigación Acción Participativa (IAP) (...) exige también por parte del investigador características tales como; empatía, liderazgo y previsión, las cuales son realmente necesarias para 
cualquier profesional de la enseñanza".

A criterio de la investigadores, la problemática estaría centrada en la demanda insatisfecha de una orientación universitaria dirigida hacia lo social pero centrada en la ética y lo técnico, porque si no se produce el compromiso social por parte de todos los sujetos significantes que conforman la universidad, estos desde el punto de vista ético, estarían sirviendo solamente para fortalecer el estado injusto de cosas; mientras que desde el punto de vista técnico, sin la pertinencia social, la educación universitaria se vuelve inoperante, irrelevante, repetitiva, vieja y, en el mejor de los casos, abstracta. En otras palabras, en los estudiantes llamados a ejercer la inclusión universitaria no existe conciencia acerca de que la acción universitaria implica no sólo transmitir métodos, conocimientos, habilidades, sino también hermanarse con el compromiso social que despliega en el saber ser y el saber vivir con otros. De manera que no asumen que la educación universitaria no sólo tiene que ver con la transmisión de saberes, lo que resulta evidente, sino también con el cultivo de la capacidad crítica, con la creación de actitudes nuevas y mejores. Sin duda alguna, lo anteriormente presentado contradice lo señalado por López (2019: 84), al enunciar el papel de la universidad hoy:

La universidad como entidad docente e investigadora es el principal agente de cambio que debe proporcionar respuestas a los problemas de la sociedad, reconociendo la crisis de la civilización, producto del modelo depredador capitalista, identificando dialécticamente las diversas fuerzas que inciden en el deterioro progresivo del planeta.

Por consiguiente, el problema no es solo del estudiante, es del docente, representante de la Universidad, quien no percibe y comprende la interacción comunitaria como un ente transformador. De allí que surja la relevancia de abordar el compromiso social tanto en autoridades, docentes, administrativos, obreros, docentes como en estudiantes de la Universidad Nacional Experimental Simón Rodríguez Núcleo Coro, por cuanto esto permite entender la visión con la cual se hace interacción comunitaria, existiendo brechas entre generar una gestión del conocimiento basado en responsabilidad social trasformadora y el trabajo asistencialista que aun predomina en 


\section{CIENCIAMATRIA \\ Revista Interdisciplinaria de Humanidades, Educación, Ciencia y Tecnología \\ Año V. Vol. V. №9. Julio - Diciembre 2019 \\ Hecho el depósito de ley: pp201602FA4721 \\ ISSN-L: 2542-3029; ISSN: 2610-802X \\ Universidad Nacional Experimental Francisco de Miranda (UNEFM). Santa Ana de Coro. Venezuela}

Marelis Castro; Josía Isea

las universidades y específicamente no escapa la Universidad Nacional Experimental Simón Rodríguez, Núcleo Coro, donde se circunscribe el estudio.

En complemento de lo expuesto, pareciera existir un vacío epistémico - teórico sobre la interacción comunitaria y la responsabilidad social universitaria en los anales de la bibliografía referente al tema, siendo esta una oportunidad para generar una teoría emergente en donde se proyecte desde las vivencias de los actores sociales, la construcción de elementos epistémicos emergentes sobre el fenómeno de estudio, siendo en este caso la interacción comunitaria como arista integradora entre la universidad y la sociedad venezolana.

Desde lo planteado, los investigadores se proponen generar las bases epistémicas emergentes para configurar un aporte teórico que oriente a los actores en realizar tanto la interacción comunitaria como el servicio comunitario a la gestación del conocimiento desde ambos entes con el fin de promover desde la universidad, una vinculación en concordancia con los nuevos paradigmas de la extensión universitaria. Por consiguiente, se plantean la siguiente interrogante de investigación:

¿Cómo construir una episteme teorética que fundamente la responsabilidad social en pro de la función interacción comunitaria en contextos universitarios: Caso de Estudio la Universidad Nacional Experimental Simón Rodríguez? Dar respuesta a la precitada pregunta, es tarea ardua y apasionante que pasa por conocer lo conocido en el entendido que nada surge de la nada, que existen conocimientos previos relevantes que dan soporte a uno nuevo construyendo un pensamiento evolutivo enriquecido en el ámbito de estudio para luego ir ahondando en la realidad desde un paradigma constructivista (Guba y Lincoln, 1990), con apoyo de la teoría fundamentada expresada por Strauss y Corbin (1990).

\section{Propósito de la investigación}

Construir una episteme teorética que fundamente la responsabilidad social en pro de la interacción comunitaria en contextos universitarios: Caso de Estudio la Universidad Nacional Experimental Simón Rodríguez 


\section{DESARROLLO}

\section{Comprensión Epistémica de la Investigación}

Las vías para la generación de conocimiento científico o enfoques epistémicos sustentan los llamados paradigmas de investigación científica que responden ellas y que se caracterizan en función de las razones básicas del conocimiento, su postura ontológica, enfoque epistemológico y estrategia metodológica.

En función de esto, la comprensión epistémica de esta investigación pasa por asumirse en el paradigma o perspectiva teórica constructivista donde, de acuerdo a Latorre, Del Rincón y Arnal (1996), la naturaleza de la realidad a investigar es dinámica, múltiple, holística, construida, global, que se aprehende en un proceso de interacción con la misma, donde se reivindica la propia experiencia humana como fuente de conocimiento, el objeto a estudiar también es observador participante, hay influencia de los valores y su propósito es comprender e interpretar la realidad o generar teoría.

Así, los investigadores apoyados en las teorías referenciales, interactuaron con facilitadores y autoridades de la UNESR (Universidad Nacional Experimental Simón Rodríguez), entrevistándolos a profundidad y categorizando las unidades de análisis discursivas, comparando e interpretando la opinión que tenían sobre la temática abordada, develando así nueva teoría puesto que la realidad no puede explicitarse sino es a través de la interpretación que los sujetos hacen de la situación en sus respectivas interacciones.

En función de lo expuesto, la metodología utilizada fue la cualitativa, situando las cuestiones referidas a las distintas vías o formas de investigación en torno a la realidad, por lo tanto, la tarea, de acuerdo A este respecto Taylor y Bogdan (1996) se refieren a la investigación cualitativa como aquella que se realiza desde dentro de la situación estudiada, captando una imagen fiel de lo que dicen y hacen las personas, se deja que las palabras y las acciones hablen por sí mismas. 


\section{Sujetos significantes a la Investigación}

En cuanto a los sujetos significantes a la investigación, Gómez (2006:6) expresa que la selección de los informantes en relación a calidad y cantidad "es difícil determinar a cuántas personas se debe entrevistar en los estudios cualitativos. Algunos investigadores tratan de entrevistar a los que realmente aportan información relevante a la temática"

En el caso del presente estudio se escogieron como sujetos significantes, cinco (5) facilitadores y dos (02) autoridades universitarias en forma deliberada, intencionalmente, los mismos corresponden a facilitadores de planta con categoría de ordinarios y dedicación de tiempo completo y exclusiva por considerar que ser los que mayor permanencia tienen en la institución (UNESR).

Debe acotarse que se consideró el criterio de selección de docentes y autoridades universitarias, en virtud de la capacidad de aportar información significativa, necesaria y requerida en cuanto a la descripción e interpretación de la dinámica educativa que dichos actores realizan.

\section{METODOLOGIA PARA LA RECOLECCIÓN E INTERPRETACIÓN DE LA INFORMACIÓN}

En cuando al proceso de investigación desarrollado desde una perspectiva metodológica en esta investigación se procesó del siguiente modo:

a) Para el momento teórico y metodológico del estudio, se emplearon los protocolos instrumentales y técnicas de la investigación documental: Búsqueda de fuentes vía Internet, observación documental, resúmenes analíticos, fichaje bibliográfico, elaboración de cuadros, gráficos y otros inherentes a la misma.

b) Técnica de la entrevista en profundidad: Martínez (2000), le da gran relevancia por ser el instrumento técnico que presenta sintonía epistemológica con este enfoque etnográfico. Es flexible, dinámica, no directiva. El entrevistador invita al entrevistado a hablar, sin contradecirle ni discutir; se encarga de mantener la conversación.

En la entrevista se utilizó una guía contentiva de planteamientos generales con base a 
las categorías del estudio, estructuradas en cinco (05) preguntas que permitieron respuestas abiertas dadas según el pensamiento de los participantes, su conocimiento y sentimientos, para lo cual acota Gómez (ob. cit.:4), "no es un protocolo estructurado.

Se trata de una lista de áreas generales que deben cubrirse con cada informante (...) La guía de la entrevista sirvió solamente para recordar que se debe hacer preguntas sobre ciertos puntos". Esta estuvo respaldada con el uso del apunte de notas relevantes al contexto y actuación del informante clave, las mismas sirvieron de refuerzo.

En síntesis, la entrevista se concibió y desarrolló como un encuentro cara a cara, entre los investigadores y los informantes, no directiva, orientada con el apoyo de un guion y dirigida a interpretar los constructos teóricos que poseen los sujetos significantes al estudio sobre la generación de una responsabilidad social para la función interacción socio comunitaria en contextos universitarios. Caso de estudio: UNESR.

c) La Técnica de la Observación Participante: Es la técnica más usada para adquirir información; se concibe como la estrategia de investigación que supone la interacción social entre los investigadores y los sujetos significantes de estudio en el contexto de estos últimos y durante la cual se recogerá información de modo sistemático, tratando de no ser intrusivo y respetando el principio de proximidad. Para ello el investigador interactúa, forma parte activa con el grupo a investigar (Martínez, 2000).

En este marco metódico, el presente se apoyó en la toma de notas o anotaciones de campo, recabando datos sobre la dinámica para la generación de una responsabilidad social para la función interacción socio comunitaria en contextos universitarios.

\section{Rigor de la Investigación: Validez y Fiabilidad}

El juicio de expertos es un método de validación útil para verificar la fiabilidad de una investigación que se define como "una opinión informada de personas con trayectoria en el tema, que son reconocidas por otros como expertos cualificados en éste, y que pueden dar información, evidencia, juicios y valoraciones" (Martínez, 2000: 29).Tras someter un instrumento de cotejo a la consulta y al juicio de expertos éste ha de reunir dos criterios de calidad: validez y fiabilidad. 


\section{CIENCIAMATRIA \\ Revista Interdisciplinaria de Humanidades, Educación, Ciencia y Tecnología \\ Año V. Vol. V. №9. Julio - Diciembre 2019 \\ Hecho el depósito de ley: pp201602FA4721 \\ ISSN-L: 2542-3029; ISSN: 2610-802X \\ Universidad Nacional Experimental Francisco de Miranda (UNEFM). Santa Ana de Coro. Venezuela}

Marelis Castro; Josía Isea

Pérez (1994), se refiere a los procesos de triangulación y saturación como instrumentos de validación que permiten aislar los sesgos e imprecisiones que puedan producirse con la aplicación de una sola técnica. Debe acotarse, que el término triangulación se deriva de las técnicas de reconocimiento del terreno, en donde se trazan líneas de al menos dos hitos, en distintas direcciones y se encuentra su punto de intersección (Patton, 1990).

Un problema puede ser investigado a fondo sólo cuando la información recolectada proviene de más de una fuente, caso del presente estudio, cuando se utiliza más de un método o herramienta de investigación o cuando más de un investigador (con perspectivas diferentes) participan en el estudio.

Para este estudio se utilizó la triangulación entre diferentes fuentes de datos a saber: Sujetos significantes, teorías de entrada y subjetividades de los investigadores; determinándose luego las intersecciones o coincidencias que surgieron a partir de las diferentes apreciaciones.

La información obtenida mediante la observación participativa de los investigadores, fue cruzada con la información procedente de la entrevista a profundidad a fin de aportar los elementos para la interpretación de los constructos que poseían los docentes y participantes, para así configurar la teoría de salida o estructura teórica emergente.

\section{Procesamiento y Análisis de la Información}

La información derivada de la investigación de campo, obtenida mediante la aplicación de las entrevistas en profundidad y las observaciones se interpretó en función del propósito central de la investigación y del marco teórico referente. Es importante señalar que se siguió un enfoque emergente durante el procedimiento investigativo, por cuanto, en el marco del enfoque cualitativo que sustenta esta disertación.

Las fases interactuaron, en oportunidades, de forma paralela o multidireccional, en un continuo ir y venir entre los supuestos teóricos y la experiencia de la autora, lo que generó un continuo proceso dialógico y un transitar dinámico sensible a las particularidades de cada unidad de análisis suministrada por los sujetos significantes. 
Latorre; Del Rincón y Arnal (1996), señalan que esta parte del trabajo constituye uno de los momentos más importantes del proceso de investigación e implico trabajar la data, recopilarla, organizarla en unidades manejables, sintetizada, buscar regularidades o modelos entre ellas, descubrir qué era importante y qué aportaba a la investigación. Coinciden con esta idea, los aportes de Guba y Lincoln (1990), quienes plantean que el sentido del análisis de datos en la metodología cualitativa consiste en reducir, categorizar, clasificar, sintetizar y comparar la información con el fin de obtener una visión lo más completa posible de la realidad en estudio y realizar el proceso de análisis sistemática y ordenadamente, aunque no rígido.

Conviene destacar lo señalado por Buendía; Colás y Hernández (1998), los cuales señalan que la exposición de la información de manera sistemática y visual es una actividad importante en el análisis de los datos; las formas más comunes que se utilizan son las matrices y los diagramas.

\section{Síntesis conceptual}

En el siguiente apartado, se procedió en la conformación del proceso interpretativo analítico; en ese sentido, la Teoría se incorporó como la técnica de análisis de la investigación. Bajo dicha mirada, la teoría emerge desde los datos, Straus y Corbin (2002), pues es una metodología que tiene por objeto la identificación de procesos sociales básicos como punto central de la teoría.

A través de esta metodología se pueden descubrir aquellos aspectos que son relevantes de una determinada área de estudio. La Teoría Fundamentada utiliza una serie de procedimientos que, a través de la inducción, genera una teoría explicativa de un determinado fenómeno estudiado.

Al respecto, los conceptos y las relaciones entre los datos son producidos y examinados continuamente hasta la finalización del estudio. Así, Strauss y Corbin (2002) aseguran que si la metodología se utiliza adecuadamente reúne todos los criterios para ser considerada rigurosa como investigación científica.

La Teoría Fundamentada permite construir teorías, conceptos, hipótesis y 
Marelis Castro; Josía Isea

proposiciones partiendo directamente de los datos y no de los supuestos a priori, de otras investigaciones o de marcos teóricos existentes. La teoría generada se desarrolla inductivamente a partir de un conjunto de datos. Si se hace adecuadamente, esto significa que la teoría resultante coincidirá al final con la realidad objeto de estudio.

En esa dirección, el procedimiento de reducción de datos se desarrolló por la vía inicial de la codificación abierta, con la técnica del análisis de contenido, para avanzar en la agrupación informativa en un sistema categorial y sub categorial, que empleó como método la comparación constante, y así se estableció un ordenamiento informativo axial. Desde allí, se transitó en elementos teóricos sustantivos constituidos desde las conceptuaciones emergentes, con lo cual, su integración arrojó conceptos abarcadores que derivaron en una aproximación de teoría formal.

En el estudio, la codificación abierta consistió básicamente en separar, frase por frase, el texto de las unidades de análisis y las entrevistas de los informantes clave sobre sus experiencias, mediante la técnica que los autores denominan rotulación; a fin de codificar segmentos de texto referidos a un mismo tema. Así, se optó por asociar cada concepto obtenido en negrillas, correspondiente con una categoría concreta, así como también a las notas o memorandos, que sirvieron para facilitar la posterior descripción de las categorías obtenidas por cada tipo de unidad de estudio. 
CIENCIAMATRIA

Revista Interdisciplinaria de Humanidades, Educación, Ciencia y Tecnología

Año V. Vol. V. №9. Julio - Diciembre 2019

Hecho el depósito de ley: pp201602FA4721

ISSN-L: 2542-3029; ISSN: 2610-802X

Universidad Nacional Experimental Francisco de Miranda (UNEFM). Santa Ana de Coro. Venezuela

Marelis Castro; Josía Isea

\section{Codificación Axial}

\section{Diagrama 1.- ¿Cómo generar la Responsabilidad Social para la Interacción Comunitaria en Contextos Universitarios?}

\begin{tabular}{|c|c|c|c|c|}
\hline $\begin{array}{l}\text { DIAGNOSIS Y } \\
\text { AUTOREVISION }\end{array}$ & $\begin{array}{c}\text { INTERACCIÓN } \\
\text { COMUNITARIA } \\
\text { INTEGRATIVA } \\
\text { E } \\
\text { INTEGRADORA }\end{array}$ & $\begin{array}{c}\text { TRANSFORMACIÓN } \\
\text { SOCIALISTA } \\
\text { UNIVERSITARIA }\end{array}$ & $\begin{array}{c}\text { IMBRICACIÓN } \\
\text { COMUNITARIA } \\
\text { MULTIDISCIPLINARIA }\end{array}$ & $\begin{array}{c}\text { PRAXIS } \\
\text { EDUCATIVA } \\
\text { COMPROMETIDA } \\
\text { DESDE EL AMOR }\end{array}$ \\
\hline $\begin{array}{l}\text { Universidad como } \\
\text { institución gestora, } \\
\text { Compromiso hacia } \\
\text { el desarrollo social, } \\
\text { Vinculación social } \\
\text { del colectivo, } \\
\text { universitario, } \\
\text { Pertinencia social } \\
\text { del Curriculum } \\
\text { Vincular al sector } \\
\text { educativo para } \\
\text { generar la } \\
\text { interacción social y } \\
\text { la interacción } \\
\text { comunitaria } \\
\text { Concienciación del } \\
\text { colectivo } \\
\text { Concientización en } \\
\text { valores } \\
\text { Formación en } \\
\text { valores } \\
\text { Respeto como } \\
\text { punto primigenio } \\
\text { Concientizar los } \\
\text { valores } \\
\text { comunitarios } \\
\text { Corresponsabilidad }\end{array}$ & $\begin{array}{l}\text { Integración de la } \\
\text { Interacción } \\
\text { Comunitaria } \\
\text { dimensionalmente, } \\
\text { Imbricación } \\
\text { Universidad - } \\
\text { Comunidad, } \\
\text { Principios de } \\
\text { horizontalidad y } \\
\text { participación, } \\
\text { Planeamiento } \\
\text { estratégico } \\
\text { Generación } \\
\text { espacios critico } \\
\text { reflexivos } \\
\\
\text { Trascendencia del } \\
\text { claustro } \\
\text { universitario }\end{array}$ & $\begin{array}{l}\text { Un nuevo ser social } \\
\text { dentro del contexto de } \\
\text { un nuevo paradigma, } \\
\text { Concepción del } \\
\text { crecimiento humano } \\
\text { para una nueva } \\
\text { interacción comunitaria, } \\
\text { Autosuficiencia } \\
\text { Comunitaria para el } \\
\text { bienestar social, } \\
\text { Una interacción que } \\
\text { suponga cambios a } \\
\text { nivel de la universidad y } \\
\text { a nivel de la } \\
\text { comunidad. } \\
\text { Las distintas áreas y } \\
\text { facultades sirvan de } \\
\text { plataforma desarrollo } \\
\text { comunitario } \\
\text { Educación permanente } \\
\text { para la responsabilidad } \\
\text { social con énfasis en lo } \\
\text { comunitario. }\end{array}$ & $\begin{array}{c}\text { Enfoque } \\
\text { humanista } \\
\text { constructivo } \\
\text { Reconocimiento } \\
\text { a la otredad } \\
\text { Pertinencia } \\
\text { social del } \\
\text { Curriculum, } \\
\text { Trabajo en red } \\
\text { comunitaria de } \\
\text { acción } \\
\text { universitaria, }\end{array}$ & $\begin{array}{c}\text { Accionar } \\
\text { comprometido } \\
\text { Equidad } \\
\text { Ejemplaridad } \\
\text { Dialógica } \\
\text { Práctica educativa } \\
\text { democrática, } \\
\text { andragógica } \\
\text { Carácter educativo } \\
\text { sociocomunitario } \\
\text { Reforzamiento de la } \\
\text { ética y valores con } \\
\text { estrategias. } \\
\text { Preparar planes y } \\
\text { proyectos que } \\
\text { generen esfuerzo del } \\
\text { estudiante para el } \\
\text { desarrollo comunitario, } \\
\text { Servicio Estudiantil } \\
\text { Comunitario sostenible } \\
\text { y sustentable. }\end{array}$ \\
\hline \multicolumn{2}{|c|}{$\begin{array}{l}\text { RESPONSABILIDAD SOCIAL CRÍTICA } \\
\text { REFLEXIVA }\end{array}$} & $\begin{array}{c}\text { INTERACCIÓN } \\
\text { COMUNITARIA DE } \\
\text { MARCADO } \\
\text { CARÁCTER } \\
\text { SOCIALISTA }\end{array}$ & \multicolumn{2}{|c|}{$\begin{array}{l}\text { PRAXIS EDUCATIVA COMPROMETIDA CON EL } \\
\text { ACCIONAR COMUNITARIO }\end{array}$} \\
\hline
\end{tabular}

Los investigadores (2018) 
Marelis Castro; Josía Isea

\section{Memo Descriptivo de la Codificación Axial}

En el diagrama que se desprende como resultado de la codificación axial, se aprecia que generar la Responsabilidad Social para la Interacción Comunitaria en contextos universitarios, parte de una Diagnosis y autorevision de la universidad como institución gestora para generar un compromiso hacia el desarrollo social del colectivo universitario, con pertinencia social del currículo vinculados al sector educativo para generar la interacción social y la comunitaria, generando así, la formación y concienciación en valores comunitarios con corresponsabilidad.

Ahora bien, la interacción comunitaria integrativa e integradora, dimensionalmente imbrica a la universidad-comunidad, con principios de horizontalidad y participación, que partan de un planeamiento estratégico que permita generar espacios donde la crítica constructiva trascienda del claustro universitario.

Cabe destacar, que la transformación socialista universitaria parte por transformar un nuevo ser social dentro del contexto de un nuevo paradigma, para fomentar el crecimiento humano una nueva interacción comunitaria para el desarrollo de la autosuficiencia comunitaria, para el bienestar social. Una interacción que suponga cambios a nivel de la universidad y a nivel de la comunidad, es decir, una educación permanente para la responsabilidad social con énfasis en lo comunitario.

Es por esto, que la universidad en un enfoque humanista constructivo debe generar la imbricación comunitaria interdisciplinaria bajo el principio de la otredad el trabajo en red comunitaria de acción universitaria dentro de un currículo totalmente pertinente.

En este mismo contexto, trabajar el contacto humano para establecer una base sólida desde la praxis educativa para la formación a lo largo de toda la vida, se hace necesario un accionar comprometido en la equidad, ejemplaridad, dialógica, practica educativa democrática y andrológica, con énfasis en el reforzamiento de la ética, la universidad como gestora responsable debe preparar planes y proyectos que generen esfuerzo del estudiante para el desarrollo comunitario, el servicio estudiantil sostenible y sustentable. 


\section{Corpus Teórico Emergente}

El proceso recursivo del pensar y repensar las ideas; la organización y reorganización del pensamiento desde un enfoque constructivista, para reubicar el pensamiento en el fenómeno estudiado: la responsabilidad social para la función interacción socio comunitaria en contextos universitarios y dar respuesta a la interrogante central de estudio como lo es ¿Qué categorías de análisis sustentan un corpus teórico para la generación de una responsabilidad social para la función interacción socio comunitaria en contextos universitarios?, buscando lograr unificar la visión con la de los informantes para lograr integrar estas ideas a las teorías de apoyo en contraste con la percepción de la realidad de los investigadores, llevan a las reflexiones que se presentan a continuación, en un intento de sintetizar los hallazgos desde una hermeneusis integradora para lograr generar nuevos constructos sobre las categorías abordadas en el estudio.

La teoría emergente desde su supuesto ontológico, pretende aportar ideas y planteamientos que generen la responsabilidad social para la interacción comunitaria en contextos universitarios, cuestión tan urgida en la sociedad venezolana. En lo referente a lo epistemológico, se asume una mirada constructivista integradora, con una realidad percibida multidimensionalmente.

De allí pues que se parte por señalar que la educación universitaria se constituye en eje formativo central para la nueva sociedad, al sustentar la formación de los hombres y mujeres profesionales con pertinencia social, con responsabilidad en la consecución de transformaciones de la sociedad. La Universidad y sus procesos formativos constituyen un todo, cuya alta responsabilidad está centrada en un accionar comprometido para formar al talento humano preparado para tomar las riendas de la sociedad y las decisiones que mejor satisfagan las necesidades de su entorno social.

De allí, que dentro de los presupuestos implícitos en las concepciones que en ella debe prevalecer, se destaca la presencia de una perspectiva epistemológica que comprende a la sociedad como una construcción colectiva de las personas que la conforman, a su vez influidas por esa construcción, susceptible de crítica y de cambios; por otro lado, las 


\section{CIENCIAMATRIA \\ Revista Interdisciplinaria de Humanidades, Educación, Ciencia y Tecnología \\ Año V. Vol. V. №9. Julio - Diciembre 2019 \\ Hecho el depósito de ley: pp201602FA4721 \\ ISSN-L: 2542-3029; ISSN: 2610-802X \\ Universidad Nacional Experimental Francisco de Miranda (UNEFM). Santa Ana de Coro. Venezuela}

Marelis Castro; Josía Isea

relaciones entre todo el colectivo universitario y los integrantes de la comunidad poseen un carácter dialéctico, materializadas en la búsqueda de encuentros cotidianos, que permitan también acceder a las visiones de mundo de los involucrados; y, finalmente, la génesis de las prácticas sociales es comprendida no desde una causalidad mecánica del entorno universidad - comunidad, sino más bien desde la relación existente entre las disposiciones de los agentes sociales y las posibilidades y censuras del entorno.

Rodríguez (2012), señala que la responsabilidad social universitaria, entendida como proyección social, extensión universitaria o servicio social, entraña una realidad como consecuencia inevitable de la enseñanza-aprendizaje y la investigación y responde al principio universal de hacer el bien y compartirlo con los demás.

Se aspira comprender que la responsabilidad social desde contextos universitarios como servicio para la sociedad, es fruto de una gestión basada en la ética y la eficiencia. De ahí que la Responsabilidad Social por parte de la universidad, pueda entenderse como una vocación que trasciende la esfera del conocimiento y la investigación para concretarse en la utilidad de la sociedad.

En este marco, generar la responsabilidad social para la interacción comunitaria en contextos universitarios pasa por reconocer como núcleos del saber o ejes articulantes: La necesidad que la universidad asuma una seria posición critico - reflexiva de su accionar en pro de un desarrollo social, para luego gestar una verdadera transformación de marcado carácter socialista desde una praxis educativa socio implicadora y comprometida con el accionar comunitario.

Desde el eje o núcleo de la Responsabilidad social crítica - reflexiva, se asumen dos grandes categorías como son la Diagnosis y auto revisión y la interacción comunitaria integrativa e integradora.

La primera caracterizada por los rasgos: Universidad como institución gestora, Compromiso hacia el desarrollo social, Vinculación social del colectivo, universitario, Pertinencia social del Curriculum Vincular al sector educativo para generar la interacción social y la interacción comunitaria, concienciación del colectivo, concientización en valores, formación en valores, respeto como punto primigenio, 
Marelis Castro; Josía Isea

concientizar los valores comunitarios y asumir la ccorresponsabilidad en el desarrollo social.

La diagnosis y auto revisión, ayuda a obtener una especie de imagen acerca de cómo está la universidad con relación a los impactos que gesta en pro del desarrollo social desde la interacción comunitaria accionada; también ayuda a establecer las líneas de acción rectoras para la consolidación de todo un colectivo institucional y comunitario.

Con relación a la interacción comunitaria integrativa e integradora, conlleva los rasgos relativos a que dicha Interacción dimensionalmente, imbricación Universidad Comunidad, principios de horizontalidad y participación, planeamiento estratégico, generación espacios critico reflexivos y sobre todo que la universidad implemente mecanismos capaces de trascender el claustro universitario.

En lo que atañe al segundo núcleo o eje articulante, la interacción comunitaria de marcado carácter socialista, este se categoriza con énfasis en la tan necesaria transformación socialista universitaria, gestando desde el contexto universitario un nuevo ser social dentro del marco de un nuevo paradigma, con una concepción del crecimiento humano para una nueva interacción comunitaria, con el trabajo en red comunitaria de acción universitaria, buscando la autosuficiencia Comunitaria para el bienestar social, y una interacción que suponga cambios a nivel de la universidad y a nivel de la comunidad.

En este sentido y como se decía anteriormente, se precisa del tercer eje o núcleo del saber cómo lo es la praxis educativa comprometida con la interacción comunitaria, comprendiendo y asumiendo que todo accionar debe girar en pro de una pertinencia social fomentando una gestión universitaria responsable, vinculada al ser y al hacer en comunidad. Paralela a esta pertinencia, se establece la omnipresencia de lo comunitario en lo curricular, sustentada en una práctica sinérgicamente gestada dese la dimensión comunitaria.

Igualmente, el hecho investigativo comunitario, la gestión de la investigación con pertinencia social, asumiendo como plataformas las distintas áreas, facultades, departamentos, servicios para un comprometido desarrollo comunitario, se hace vital 


\section{CIENCIAMATRIA \\ Revista Interdisciplinaria de Humanidades, Educación, Ciencia y Tecnología \\ Año V. Vol. V. №9. Julio - Diciembre 2019 \\ Hecho el depósito de ley: pp201602FA4721 \\ ISSN-L: 2542-3029; ISSN: 2610-802X \\ Universidad Nacional Experimental Francisco de Miranda (UNEFM). Santa Ana de Coro. Venezuela}

Marelis Castro; Josía Isea

Cabe destacar, que no es sólo deber del docente o del estudiantado la interacción comunitaria sino que todo miembro universitario debe estar llamado a ser promotor social, y para ello se debe trascender del claustro universitario a través de la instrucción preparando planes y proyectos que generen esfuerzo de todo el colectivo para el desarrollo comunitario.

En síntesis, todo un colectivo universitario respondiendo a la misión de la naturaleza de la universidad debe sentir la responsabilidad de contribuir concretamente al progreso de la sociedad en la que opera: podrá buscar la manera de hacer más asequible la interacción comunitaria socio implicadora a todos los que puedan beneficiarse de ella. Además, tiene la responsabilidad de ayudar a promover el desarrollo social.

De allí pues que toda reflexión se inscribe, en presentar un nuevo mapa cognitivo, un corpus teórico para una realidad posible en para la generación de una responsabilidad social para la función interacción socio comunitaria en contextos universitarios con base en la razón creática y la razón pragmática del conocimiento construido, pero de modo especial a la construcción de un equilibrismo entre las dos razones del conocimiento para abordar una realidad y transformarla.

\section{REFERENCIAS CONSULTADAS}

1. ARIAS, Enith; SARMIENTO, Deyamira. RESPONSABILIDAD SOCIAL EMPRESARIAL EN FALCÓN. UN ANÁLISIS DE CONTENIDO. Revista Arbitrada Interdisciplinaria Koinonía, [S.I.], v. 1, n. 1, p. 22-42, feb. 2017. ISSN 2542-3088. Disponible $<$ http://fundacionkoinonia.com.ve/ojs/index.php/revistakoinonia/article/view/13>

2. Guba. y Lincoln, I. (1999). Fundamentos del paradigma constructivista. España: Sevilla.

3. Herrera, A. (2009) Referentes internacionales. Reflexión y acción. Informe GUNI reunión de la Red Nacional de Extensión.

4. Isea Argüelles, J., Briceño, R., \& Mayorga, T. (2019). EXPERIENCIA DE RESPONSABILIDAD SOCIAL UNIVERSITARIA DESDE LA INVESTIGACIÓN ACCIÓN - PARTICIPANTE. CIENCIAMATRIA, 2(2), 22-43. Recuperado a partir 
de http://www.cienciamatriarevista.org.ve/index.php/cm/article/view/50

5. Isea, J. (2018). La Acción Social Universitaria Venezolana. Un Camino de integración con las Comunidades. Santa Ana de Coro: Editorial Fundación Koinonía.

6. Latorre, A., Del Rincón, D., y Arnal, J. (1996). Bases metodológicas de la Investigación educativa. Barcelona: Gr92.

7. López Echegarai, M. (2019). Política de sustentabilidad ambiental. Avances en el contexto universitario. Revista Arbitrada Interdisciplinaria Koinonía, 4(7), 59-87. doi:http://dx.doi.org/10.35381/r.k.v4i7.194

8. Martínez, M. (2000) La investigación cualitativa etnográfica en educación. Manual teórico - práctico. México: Trillas.

9. Patton, M. (1990) Métodos cualitativos de investigación y evaluación. Beverly Hills, CA: Sage.

10. Pérez, S., G. (1994). La Investigación Cualitativa: retos e interrogantes. Madrid: Edit. La Muralla.

11. Rodríguez, J. (2012). Aproximación a un modelo teórico de participación social que fortalezca la calidad de vida desde el contexto de la educación venezolana. Tesis Doctoral. Universidad de Córdoba, España.

12. Strauss, A. y Corbin, J. (1990). Bases de la Investigación Cualitativa: Técnicas y procedimientos de la Teoría Fundamentada. London: Sage.

13. Taylor, S y Bodgan, R (1996) Introducción a los Métodos Cualitativos de la Investigación. La Búsqueda de Significados. Barcelona: Paidos.

C2019 por los autores. Este artículo es de acceso abierto y distribuido según los términos y condiciones de la licencia Creative Commons Atribución-NoComercial-Compartirlgual 4.0 Internacional (CC BY-NC-SA

4.0) (https://creativecommons.org/licenses/by-nc-sa/4.0/). 